the syndrome are variable, leading to delayed or missed diagnosis with potential fatality from hypoventilation and cardiorespiratory arrest.

Genetic testing of candidate genes in 15 children with ROHHAD syndrome. PHOX2B sequencing, the disease-causing gene in congenital central hypoventilation syndrome, revealed no mutations, demonstrating that late-onset ROHHAD is distinct from the congenital syndrome (Ize-Ludlow D, Gray JA, Sperling MA, et al. Pediatrics 2007 Jul;120(1):e179-88). In addition to hyperphagia and obesity, respiratory alveolar hypoventilation, thermal and other autonomic dysregulation, patients with ROHHAD have hyperprolactinemia, diabetes insipidus, and tumors of neural crest origin. The report of narcolepsy as a manifestation of this mainly respiratory syndrome may lend support to the theory of an autoimmune-mediated process as a possible cause of ROHHAD (Mahlios J, et al. The autoimmune basis of narcolepsy. Curr Opin Neurobiol 2013 Oct;23(5):767-73).

\title{
NARCOLEPSY/CATAPLEXY AND OCCULT NEUROBLASTOMA
}

Investigators at the University of Chicago and Northwestern University, Chicago, IL; University Hospital Southampton, UK; and Kiev Paediatric Hospital, Ukraine, report three children with narcolepsy and cataplexy subsequently diagnosed with neuroblastoma. Patient 1, a 3-year-old girl with gross motor delay and hypersomnia developed loss of tone and falling induced by laughter. Multiple sleep latency tests suggested narcolepsy. Six months later she became ataxic and neurologic examination revealed opsoclonus and divergent strabismus. MRI of the brain was normal, while CT scan of the chest demonstrated a $10 \times 3 \mathrm{~cm}$ left paraspinal mass, T9 to T12. Surgical resection of a ganglioneuroblastoma, and treatment with adrenocorticotropin, plasmapheresis, iv immunoglobulin, and cyclophosphamide were of no benefit. After 2 years she developed central hypoventilation syndrome requiring continuous mechanical ventilation. Patient 2, a 2-year-old girl developed narcolepsy/cataplexy followed by muscle fatigue, ataxic gait and ptosis. A diagnosis of Lambert-Eaton myasthenic syndrome prompted a search for an occult neoplasm, and MRI of chest revealed a left costovertebral junction mass at T5-T9. Resection of a ganglioneuroblastoma followed by chemotherapy was followed by slow improvement and no recurrence of ptosis, weakness, hypersomnolence, or cataplexy. Patient 3, a 3-year-old girl presented with ataxic gait and frequent falls after varicella zoster. She developed narcolepsy/cataplexy with decreased CSF hypocretin level. MRI of pelvis revealed a paraspinal mass, confirmed as neuroblastoma at biopsy. Treatment with dexamethasone and chemotherapy resulted in rapid resolution of hypersomnia and cataplexy and gradual resolution of ataxia, followed by resection of the tumor. (Sinsioco C, Silver K, Forrest KM, et al. Narcolepsy with cataplexy as presenting symptom of occult neuroblastoma. Pediatr Neurol 2013 Jul;49(1):64-7). (Response: Dr Sinsioco, University of Illinois at Chicago, $1801 \mathrm{~W}$ Taylor St, Chicago, IL 60612. E-mail: sinsioco@uic.edu).

COMMENT. Narcolepsy with cataplexy is rare in early childhood and should prompt investigation to exclude neuroblastoma. Opsoclonus, ptosis, central hypoventilation, and dysautonomia may be present but are not invariable. (Koh PS, et al. J Pediatr 1994 Nov;125(5 Pt 1):712-6). 